\title{
Rules for Plausible Reasoning
}

\author{
DOUGLAS WALTON University of Winnipeg
}

Key Words: Plausible reasoning; confidence factors; presumption; argument; linked arguments; convergent arguments; argumentation.

Abstract: This article evaluates whether Rescher's rules for plausible reasoning or other rules used in artificial intelligence for "confidence factors" can be extended to deal with arguments where the linked-convergent distinction is important.

Many of those working in the field of argumentation now accept the idea that there is a third type of reasoning distinctive from deductive and inductive reasoning called plausible reasoning, a kind of reasoning based on tentative, prima facie, defeasible weights of presumption which can be assigned to the propositions in an argument.' Some theorists have now even offered sets of rules (calculi) for plausible reasoning.

The set of rules presented by Rescher (1976) is perhaps the best known to those of us working in informal logic and argumentation. But within the field of artificial intelligence, where presumptive reasoning based on "confidence factors" is very important, e.g. in applying expert systems of technology, various proposals for rules of this type have been advanced.

This paper evaluates Rescher's rules, and one set of rules from AI (Intelliware, 1986) with a view to seeing whether or to what extent such accounts of plausible reasoning could be useful for, or adapted to, the needs of informal logic. Taking into account the vital distinction between linked and convergent arguments, new, more general rules for plausible reasoning are proposed which would be useful for evaluating argumentation in a critical discussion, in the sense of van Eemeren and Grootendorst (1984) and Walton (1992).

\section{Systems of Plausible Reasoning}

Rescher's system of plausible reasoning follows a conservative way of evaluating an argument. The least plausible proposition in a set is the weakest link in the chain of argumentation, because it represents the greatest possibility of going wrong or getting into trouble. Hence Rescher's accounts of plausible inference are generally based on the weakest link idea. It is easy to appreciate how this idea fits the context of a critical discussion.

The respondent has the obligation or function of asking critical questions in response to an argument advanced by a proponent in a critical discussion. Naturally, a critical respondent is trying to resist being persuaded by his partner's argument. He has the job of seeking out the weakest premises, and attempting to challenge or question these premises especially. This has two consequences. One is that the proponent always tries to boost up these weak premises, or potential avenues of escape (loopholes) for the respondent. The proponent always tries to have all premises as potentially being able to be backed up so that they are more plausible than the conclusion the respondent doubts or resists. But second, the respondent is always drawn towards these weakest links (loopholes) in his adversary's line of argument. So the conclusion he is supposedly being pushed towards conceding can never be rationally rated as more plausible, for him, than that weakest premise.

Another important context of application of plausible reasoning is that of deciding on a course of action based on the 
advice gathered from the solicited opinion of an expert authority on a question (Rescher, 1976, p. 6). The user interface of an expert system is designed for a very similar use. For it is the user of the system who must draw conclusions from a set of facts and rules in a knowledge base which represents the systematization of an expert's knowledge in a given domain of expertise. In using an expert system, it must be recognized that exceptions to accepted rules may exist, and therefore an approach to reasoning which assigns confidence factors (CF's) as rough guides to reliability of advice has proved most successful.

The way an expert reasons, however, in arriving at a conclusion in her field of expertise, is quite different from the way a (nonexpert) user reasons in drawing conclusions from what the expert says. The user is typically engaged in deliberating on what to do, and quite often the context is that of a critical discussion concerning the pro and contra points of view on a possible course of action being considered.2

For example, in judging the alleged fallaciousness of an argumentum ad verecundiam, the problem is typically to evaluate how an appeal to expert opinion was used in a critical discussion between two parties. ${ }^{3}$ The expert is a third party whose opinion was appealed to as a move made by one of the participants in the critical discussion. In such a case, the rules of plausible reasoning need to be formulated in the context of the critical discussion.

Although plausible reasoning involves a qualitative judgment of relative comparison of propositions, as opposed to a quantitative-numerical calculus, formalized systematization of general rules for plausible reasoning have been proposed by Rescher (1976) and other systems of rules are in use in AI programs. Among the six formal rules for plausible reasoning given by Rescher (1976, p. 15), perhaps the most fundamental and characteristic rule is the consequence condition. This condition requires that when a group of mutually consistent propositions entails a particular proposition, then the latter proposition cannot be less plausible than the least plausible proposition in the original group. This rule is also called the least plausible premise rule, and it defines the essential characteristic of plausible reasoning as a kind of logical inference, in Rescher's calculus.

In artificial intelligence, a variety of sets of different types of rules have been given, for example, in expert systems research, to provide the "inference engine" for deriving conclusions in a data base where the facts and rules lead, at best, to tentative conclusions based on degrees of confidence. In the language of $\mathrm{AI}$, a rule is a condition that may have several antecedents (premises) where the collection of antecedents is treated as a conjunction of simple propositions (facts). In one leading approach, outlined by Intelliware (1986), the rule for calculating confidence factors (CF's) for and takes the minimum plausibility value (confidence factor). Formally,

$$
\text { plaus }(\mathrm{A} \wedge \mathrm{B})=\min (\text { plaus } \mathrm{A} \text {, plaus } \mathrm{B})
$$

Then to calculate the plausibility of a conclusion based on a set of premises, we multiply the plausibility value of the rule with the plausibility value obtained from the premises (by the conjunction rule above, where there is more than one premise). Formally,

$$
\left.\begin{array}{l}
\text { plaus(conclusion) } \\
\times \text { plaus }(\text { rule }))
\end{array}=\text { plaus(premises }\right)
$$

This approach (hereafter called the product rule) is quite different from Rescher's in several important respects, most notably perhaps in allowing a plausibility value for the inference itself. And then, of course, the product rule is itself basically different from Rescher's in the specific formula of calculation used.

The basic formal rules of plausible reasoning are given by Rescher (1976, p. 15), and comparable rules for inexact inference for expert systems are given by Intelliware (1986), Main Menu, Inexact Inference, 
pp. 3-9). However, recent developments in the area of argumentation indicate two important kinds of exceptions to these rules. Accordingly, these rules need to be modified, extended and developed in new directions. The first exception concerns the distinction between two kinds of conditionals. ${ }^{4}$ In a must-conditional, 'If $\mathrm{A}$ then $B$ ' means that $B$ is true in every instance in which $A$ is true, with no exceptions. In a might-conditional, 'If $A$ then $B$ ' means that $B$ may be expected (presumed) to be true in a preponderance of typical instances in which $A$ is true. But the linkage between $A$ and $B$ is a matter of typical or customary expectation, which can admit of exceptions. The plausibility value of a must-conditional is always equal to 1 (certainty), whereas the plausibility value of a mightconditional, $v$, can range between 0 (of no value as a plausible presumption) and 1 (maximally plausible): $0 \leq v \leq 1$.

The set of rules in Rescher (1976, p. 15) is defined only for must-conditionals, but recent developments in artificial intelligence-see Forsyth (1984), Bratko (1986) and Intelliware (1986)show a clear practical need for consideration of rules of inference where "confidence factors" (certainty factors) need to be taken into account, by using inference rules with values of less than one for might-conditionals.

It has already been noted above that in Intelliware (1986) a rule (conditional proposition) can be assigned a confidence factor of less than one as a value. When inferring a conclusion from a set of premises, the way to calculate the value of the conclusion is to multiply the value of the rule (conditional) by the value of the least plausible (lowest confidence factor) premise. In Intelliware (1986, Main Menu, Inexact Inference, p. 6), the following example of calculating CF's for a single rule with a value of .60 is given. The asterisk (*) stands for multiplication (product).

Rule 1:

$$
\mathrm{CF}=0.60
$$

Stock 12 is volatile
IF

Stock 12 is hightech

$\mathrm{CF}=0.90$

AND

Stock 12 is in demand

$\mathrm{CF}=0.60$

Evaluate Rule 1:

$\mathrm{CF}($ Rule 1$)=\operatorname{Min}(0.90,0.60) * 0.60=$ 0.36

This type of rule allows us to derive conclusions using a might-conditional, or as it is called in $\mathrm{AI}$, a rule that is assigned a confidence factor of less than one $(\mathrm{CF}<1)$.

Rescher $(1977$, p. 6$)$ introduces a provisoed assertion relation, $\mathrm{A} / \mathrm{B}$, meaning that $\mathrm{A}$ ordinarily obtains provided that $\mathrm{B}$ obtains, other things being equal, which he insists (p. 7) is not to be identified with implication. However, "for simplicity" (p. 8 ), he supposes that moves in dialogue of the form A/B are "always correct," meaning that disputants can never make erroneous or incorrect claims about them. Rescher's comment (p. 8) is that this assumption "eliminates various complications" that do not matter for his present purposes. But this assumption also removes the possibility of dealing with might-conditionals by showing how to derive conclusions from them in combination with premises in plausible reasoning. What is needed is a more realistic or practical concept of frame-based conditionals (provisoed assertion relations) that are suitable to the needs of persuasion dialogue.

Might-conditionals are frame-based conditionals to the effect that if one proposition $\mathrm{A}$ is plausible, and another set of presumptions $S$ are plausible in the commitment set of a respondent, then another proposition may be presumed to have a certain weight of plausibility. For example, consider the two propositions below.

A: Jones is less than five feet tall.

B: Jones is an All-Star forward on the NBA Los Angeles Lakers.

If $\mathrm{A}$ is taken as a proposition in a commitment set of a participant in argument, then given what we all know about basketball (viz. it is practically necessary for a basket- 
ball player to be fairly tall, we would normally expect, in order to be successful as an All-Star forward on the NBA Los Angeles Lakers), then $B$ would not be plausible as a proposition in that participant's commitment set. Similarly, if $B$ were taken as a plausible presumption, by a might-conditional, it would follow that $\mathrm{A}$ would not be a plausible presumption in that same set. In fact, from the point of view of plausible argumentation, A and B are "opposites" of each other (assuming they are in the same commitment set, which also contains the set $S$ of plausible presumptions about successful players in the NBA).

In short, there is a clash or opposition between $A$ and $B$. Not a logical inconsistency, but a pragmatic inconsistency which reflects a tug of opposing plausibility weightings.

\section{Linkage of Premises in a Critical Discussion}

The second type of exception to conventional systems of plausible reasoning concerns a requirement on the linkages between pairs of premises in an argument advanced by a proponent in a critical discussion. The additional requirement needed here is that the premise-set as a whole must be taken to be plausible by the respondent to whom an argument in persuasion dialogue is directed. Otherwise, the least plausible premise rule (reflecting the conservative point of view) might fail.

This requirement of linkage of a set of premises in a useful argument in a critical discussion reflects the importance that should be placed on consistency (coherence) in a commitment set to be used as a set of premises to convince someone of a conclusion. Indeed, the primary way that interactive reasoning functions to produce maieutic insight is through the criticism of inconsistencies in an arguer's position. By dealing with the presumptive inconsistencies found by a critic, a participant in inter- active reasoning can come to a deeper understanding of his own position (commitment set).

When discussing the rules of plausible inference, we start with a set of propositions, A, B, . . . , each of which can be assigned a plausibility value. For example, the plausibility value of the proposition $A$ is written as plaus $(\mathrm{A})$. For any proposition $A$, the value of $A$ is subject to the condition: $0 \leq p \operatorname{laus}(\mathrm{A}) \leq 1$. In other words, a maximal plausibility (totally reliable) proposition can be assigned a value of 1 , and a proposition that would not count as plausible, one of no useful value to persuade a respondent of a conclusion, can be given a value of 0.5

The basic axiom of plausible inference is the consequence condition (Rescher, 1976, p. 15): when a set of mutually consistent propositions $A_{1}, \ldots, A_{n}$ implies some other proposition $\mathrm{B}$ by valid deductive argument, then the plausibility of $B$ cannot be less than the plausibility value of the least plausible proposition among the set $A_{1}, \ldots, A_{n}$. In short,

If $A_{1}, \ldots, A_{n}$ imply $B$, then plaus $(B) \geq$ $\operatorname{MIN}$ plaus $\left(A_{1}, \ldots, A_{n}\right)$

This consequence condition settles how conjunction is to be defined in plausible inference. The following plausibility rule for conjunction gives this definition. See Intelliware (Main Menu, Inexact Inference, p. 3).

$$
\operatorname{plaus}(\mathrm{A} \wedge \mathrm{B})=\mathrm{MIN}(\operatorname{plaus}(\mathrm{A}), \operatorname{plaus}(\mathrm{B}))
$$

That is, the plausibility of the conjunction $A \wedge B$ always reduces to the plausibility value of the lesser of the two propositions, A, B.

How the consequence rule determines the conjunction rule above has been shown by Rescher (1976, p. 16, theorem 3). First, recall that the following three forms of inference are deductively valid.
(I1) $\frac{\mathrm{A} \wedge \mathrm{B}}{\mathrm{A}}$
(I2) $\frac{\mathrm{A} \wedge \mathrm{B}}{\mathrm{B}}$
(13) A
$\underline{B}$ 
According to the consequence condition, the plausibility of the conclusion of a deductively valid argument must be as great as the plausibility of the least plausible premise. Since $A \wedge B$ is the only premise of (I1), it follows that the plausibility of $\mathrm{A}$ must be at least as great as that of $A \wedge B$. Similarly for (12), the plausibility of $B$ must be at least as great as that of $A \wedge B$. In other words,

(T1) $\operatorname{plaus}(\mathrm{A}) \geq \operatorname{plaus}(\mathrm{A} \wedge \mathrm{B}) ; \operatorname{plaus}(\mathrm{B}) \geq$ $\operatorname{plaus}(\mathrm{A} \wedge \mathrm{B})$

Hence whichever of $A$ or $B$ has the lesser plausibility, it still must have a value at least as great as that of $\mathrm{A} \wedge \mathrm{B}$. In other words,

(T2) MIN(plaus(A), plaus $(\mathrm{B})) \geq \operatorname{plaus}(\mathrm{A} \wedge \mathrm{B})$

But now, looking at (I3), we can see that according to the consequence condition, the plausibility of $A \wedge B$ must be at least as great as the plausibility of whichever of $A$ or B has the lesser value. In other words,

(T3) plaus $(\mathrm{A} \wedge \mathrm{B}) \geq \operatorname{MIN}($ plaus $(\mathrm{A})$, plaus $(\mathrm{B}))$

Putting (T1) and (T2) together yields the plausibility rule (T3) for conjunction given above. It has been shown then that the conjunction rule follows from the consequence condition.

So conceived, the rules for plausible inference are parallel to the rules for deductive inference. Just as conjunction was defined as a logical constant in the theory of deductive reasoning, so too conjunction will have a rule (T3) that defines it as a constant in the theory of plausible reasoning. So conceived, also, the theory of plausible reasoning presupposes the concept of deductive logical consequence that is defined in the theory of deductive reasoning. By these lights, plausible reasoning has a formal aspect which appears to make a calculus with formal rules of inference.

This parallel begins to break down, however, when certain kinds of cases of plausible reasoning enter the picture. These examples undermine the plausibility rule for conjunction, and with it, the fundamental least plausible premise rule. The latter rule states that, in a deductively valid argument (where the premises are logically consistent) the conclusion must be at least as plausible as the least plausible premise. But consider the following argument.

Case 0: (PI) Jones is less than five feet tall.

(P2) Jones is an All-Star forward on the NBA Los Angeles Lakers.

(C) Jones is a less than five-foot tall All-American forward on the NBA Los Angeles Lakers.

In this case, there may be evidence that makes (P1) highly plausible, and also other evidence that suggests that $(\mathrm{P} 2)$ is highly plausible. But although the form of argument in case 0 is deductively valid, and the premises are logically consistent with each other, the conclusion is not highly plausible. In fact, it is implausible. And since case 0 is of the form (13), the plausibility rule for conjunction also fails in case 0 .

Case 0 is a linked argument, in the sense that both premises (P1) and (P2) are required to derive (C) by a deductively valid argument form. If either of (P1) or (P2) is omitted, the argument ceases to be valid. But in some other sense perhaps, case 0 may not appear to be a linked argument, in that it would seem to be somehow characteristic of this type of argument that the line of evidence for (P1) should be separate from, or distinct from, the line of evidence for $(\mathrm{P} 2)$ and vice versa. But it does not seem obvious what "separate from" means in this context. This is a problem we return to below.

One might wonder how plausible reasoning compares to probable reasoning in this type of case. In case 0 above, part of the problem appears to be that the premises are probabilistically dependent on each 
other so that the conditional probability of either on the other is less than its unconditional probability alone. But the problem does not disappear by attempting to restrict the rules to sets of premises that are probabilistically independent of each other.

\section{Case 1: (P1) The first flip of this coin will be heads. \\ (P2) The second flip of this coin will be heads. \\ (C) Both the first and second flip of this coin will be heads.}

In this case, like the one above, the probability (or plausibility) is less than the probability (or plausibility) of the least probable (plausible) premise. Plausibility seems parallel to probability in this type of case. But, at any rate, plausibility does not follow the least plausible premise rule. And this failure is instantiated in its basic failure to follow the plausibility rule for conjunction in these cases.

Possibly to deal with this kind of exception, Rescher (1976, p. 15) adds the requirement of the compatibility condition: all propositions in a plausibility evaluation set must be "logically compatible and materially consonant with one another." To be materially consonant (footnote, p. 15) is meant "logical compatibility with certain suitable 'fundamental' stipulations of extra-logical fact." But what are these "fundamental stipulations of extra-logical fact"? Rescher does not tell us, and the resulting gap makes it hard to apply the least plausible premise rule, and to know where it is applicable to argumentation and where not. For clearly the exceptional cases above indicate that the rule is not applicable in some instances.

The third exception to the conventional rules of plausible reasoning arises through the distinction between linked and convergent arguments, now commonly used in informal logic. The exception noted in the present section arises because, in linked arguments, the premises must be connected together in such a way as to provide a plausible commitment set or position from which the respondent can be persuaded to accept a particular conclusion. In the next section, another exception arises through the fact that not all arguments advanced in persuasion dialogue are linked arguments.

In a linked argument, a bundle of premises is taken together as a fixed set representing the commitment set of a respondent at one move in dialogue. However, in dynamic interactive reasoning, "new knowledge" may be added to the commitment store of a participant in dialogue.

\section{Linked and Convergent Arguments Revisited}

The third exception concerns the distinction between two kinds of argument techniques represented in argument diagramming, namely linked and convergent arguments. Since the reader conversant with informal logic is already familiar with these techniques of argument diagramming, no further, more elaborate examples need to be presented here. It is enough to note that convergent and linked arguments can be combined into larger networks of argument structures, by means of serial connections joining subarguments together.

The basic rule of plausible reasoning in the Rescher framework, as noted, is the least plausible premise rule, which states that in a deductively valid argument, the conclusion must be as plausible as the least plausible premise. This rule works well in critical discussion for linked arguments, but not for convergent arguments. Typically, in a convergent argument, a conclusion is based on some existing evidence, but then some new and independent evidence comes along. If this new evidence is stronger than the old evidence, there should be an upgrade of the plausibility value of the conclusion, based on the value of the new premises. In such a case, if there is one "old" premise and one "new" 
premise, for example, the value of the conclusion should be set at the value of the most plausible premise-in this instance, the value of the "new" premise.

It follows that the least plausible premise rule is not universal for plausible reasoning. It fails in convergent arguments. It also fails where the linkage between premises and conclusion is that of a might-conditional.

The distinction between convergent and linked argumentation is not modelled in classical logic where, for example, we have valid forms of inference like ' $A \wedge B$, therefore $\mathrm{A}$ '; and the deduction theorem allows us to treat separate premises as a grouped conjunction of propositions in a single premise. But in a critical discussion the distinction between uses of these two types of argument is fundamental because each of them has to be defended against criticisms in a fundamentally different way.

In a linked argument, the respondent, who is inclined to be resistant to being convinced of the proponent's conclusion, will try to reject the premises if the argument is otherwise convincing. And he will seek out the weakest of the premises, for if one premise alone fails, the whole argument fails to persuade successfully. But in a convergent argument, each premise is a separate line of argument. So if one fails, the proponent can rely on the other. This fundamental difference is basic to the structure of using inference in critical discussion,

In figure 0 , there are two premises $A$ and $B$, used as a basis to support a conclusion $\mathrm{C}$.

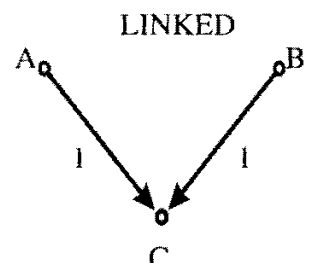

Figure 0

\section{CONVERGENT}

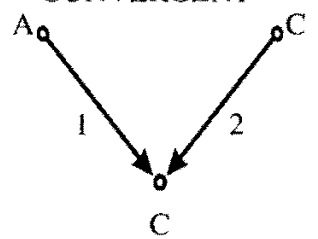

$\mathrm{C}$ gent argument each of $\mathrm{A}$ and $\mathrm{B}$ is independent of the other. ${ }^{6}$ What this means, in dialectical terms, is that the use of each type of argument has a distinctive pragmatic rationale.

This duality of pragmatic rationale was recognized and clearly stated by Windes and Hastings (1965), in their discussion of how to organize a proof when your goal is to construct a convincing case in order to persuade an audience to accept a particular proposition. Within such a context of persuasion dialogue, Windes and Hastings postulate $(1965$, p. 215) that there will be an "over-all argument" that states the issues (the global level of argumentation), and subarguments that are local contentions supporting these global issues. Serial argumentation connects some subarguments to other subarguments, resulting in extended chains of argumentation in a proof.

What is especially interesting here is that Windes and Hastings clearly distinguish between linked and convergent arguments, and articulate a basic principle of plausible inference governing each type of argument. First, they describe linked argumentation, and express what is, in effect, a statement of the weakest link principle as applicable to linked argumentation. In convincing an audience of a particular proposition, they wrote, there may be several issues, and the principle of argumentation is: "Each one of the issues must be established for the proposition to be established." (1965, p. 216) In other words, as they put it: "If any issue is not proved, then the proposition is not proved." (p. 216) They recognize, as well, that this principle of reasoning is typically embedded in a larger process of a chain of arguments that may be quite long.

This statement of Windes and Hastings expresses the basic pragmatic rationale behind linked argumentation in the context of persuasion dialogue. It expresses the idea that a linked argument is only as strong as its weakest premise. For if any

In the linked argument, both premises $\mathrm{A}$ and $B$ are needed to prove $C$. In the conver- 
premise (issue) is not proved, in a linked argument, then the conclusion is not proved. In a linked argument, the premises are interdependent, and if the audience doubts one premise, or finds it weak and unconvincing, then the audience will not be persuaded by the argument to accept its conclusion.

Windes and Hastings went on (p. 216) to recognize a second type of argumentation where there are "independent lines of reasoning" that "lead to the same conclusion," i.e. what we have called convergent argumentation.

They cite the following case, where "three reasoning processes" are used to support the conclusion, 'The corn crop of Dullnia is failing.'

Case 2: 1. Dullnia is buying corn on the world market. (Reasoning from effect to cause.)

2. The testimony of an agricultural expert who visited Dullnia. (Testimonial evidence.)

3. The presence of drought and poor growing conditions this year. (Cause to effect.)

In describing the pragmatic rationale of this type of (convergent) argument in persuasion dialogue, Windes and Hastings claim that both the number and the plausibility of the component arguments can be important (p. 217). Two other pieces of advice they offer the advocate generally - whether the argument is linked or convergent-are to use as many different lines of argument as possible, "giving precedence to the strongest proofs." (p. 218) This significant remark suggests another pragmatic rationale that (in the present author's opinion) is especially and distinctively applicable to convergent argumentation. This is the rationale, from the point of view of the advocate of a convergent argument in a persuasion dialogue, of giving precedence to the strongest line of argument, where more than one (independent) line of support for your conclusion is available.
These pragmatic rationales for linked and convergent arguments both have a dual nature, reflecting the character of persuasion dialogue. From the point of view of the proponent, or advocate of an argument, his function is to persuade the respondent by finding premises that will meet the burden of proof for that respondent. From the point of view of the respondent, his function is to critically question the premises of the proponent's arguments, finding a way to resist being persuaded, if he can.

This framework leads to the following characteristic general formulations of a pragmatic rationale and a plausibility rule for both of these types of argumentation in persuasion dialogue.

\section{PRAGMATIC RATIONALE FOR LINKED ARGUMENTATION:}

If the respondent successfully questions one premise, the whole argument fails to meets its burden of proof. So the respondent can choose to attack one or the other.

\section{PRAGMATIC RATIONALE FOR CON- VERGENT ARGUMENTATION:}

If the respondent questions one premise. the other can be brought to bear to back up the conclusion. So the respondent needs to attack both, to refute the argument. Matching each of the se pragmatic rationales is a corresponding rule for plausible reasoning.

\section{PLAUSIBILITY RULE FOR LINKED ARGUMENTS:}

$C$ has the value of the least plausibility value of the pair $(A, B)$.

\section{PLAUSIBILITY RULE FOR CONVER- GENT ARGUMENTS:}

$C$ has the value of the greater plausibility value of $(A, B)$.

From the point of view of the critical questioning of linked and convergent arguments, each type of argument has its own characteristic type of strategy as well.

\section{STRATEGY FOR QUESTIONING A LINKED ARGUMENT: \\ Generally attack the weaker (weakest) premise (other things being equal).}


STRATEGY FOR QUESTIONING A CONVERGENT ARGUMENT:

There is no point in starting by attacking the weaker premise. You might as well attack the stronger premise right away.

These differences have fundamental implications for the project of formulating rules of plausible reasoning for use in a critical discussion.

\section{New Rules for Convergent and Linked Arguments}

The basic idea of plausible reasoning has, to this point, been typified by the least plausible premise rule. This rule, it will be recalled, states that the conclusion of a deductively valid argument is at least as plausible as the least plausible premise of the argument. Now we have distinguished between linked arguments and other kinds of arguments like convergent, divergent and serial arguments. However, some important exceptions to the least plausible premise rule need to be explained. For while the least plausible premise rule holds generally for valid linked arguments at the local level, it is superseded by other rules of plausible inference in convergent arguments, and in some serial arguments.

The least plausible premise rule derives its justification from the characteristics of the critical discussion as a context of use. Generally, an argument in a critical discussion is a kind of interchange where the proponent of an argument is trying to persuade the recipient (respondent) of the argument to accept the conclusion. However, generally speaking, it is a feature of this kind of dialogue that the recipient does not accept the conclusion of the argument, at least to begin with, and he is inclined to doubt or even reject the conclusion. This being the case, the recipient of a valid argument will generally try to resist accepting the conclusion of an argument he has just been presented with, by seeking out the "weakest link" in the premises.
In a linked argument, the respondent should try to attack the weakest premise, because that will bring the whole argument down, if he can attack this one premise successfully. From the proponent's point of view, he can expect the respondent to be convinced by his argument only to the strength (weight) provided by his weakest premise. Hence the appropriate strategic presumption to gain assent in persuasion is the least plausible premise rule.

For example, suppose that Lester doubts that Nasir is a Christian, but Arlene advances the following argument.

Case 3: Nasir went to church.

If Nasir went to church then Nasir is a Christian.

Therefore, Nasir is a Christian,

If Lester does not dispute the first premise, and finds it relatively plausible, but he does dispute the acceptability of the second premise, and finds it much less plausible, how should he respond to Arlene's argument? If he is a smart and reasonable critic, he would attack the second premise, as the "weakest link," and he would not find the conclusion any more plausible than he finds the (weak) second premise, even though he may agree that the first premise is highly plausible. And it is the second premise that Arlene needs to defend.

So it can be appreciated why the least plausible premise rule is an appropriate rule of plausible reasoning in persuasion dialogue for valid linked arguments, like the one above. This argument is a linked argument because each premise fits together with the other to support the conclusion. Both premises are required to support the conclusion, and neither premise appears to render the other premise implausible for the respondent (or at least so we may presume, from what we know of the position of the respondent, on the information available to us as critics).

However, now let us contrast a case of a linked argument with a case of conver- 
gent argument. In the linked argument below, the two premises go together to support the conclusion. Whereas in the convergent argument, the second premise does not depend on the first, or vice versa. Each premise is an independent item of evidence to support the conclusion. Case 4: There is smoke coming from the
University.

If there is smoke coming from the University, then there is a fire in the University.

Therefore, there is a fire in the University.

This example is a linked argument, because each premise goes along with the other to help support the conclusion. In the linked argument, if one premise is weaker, then the conclusion is only made as plausible, through the argument, as this weaker premise. For example, in the linked argument above, if the first premise is highly plausible, but the second premise is only weakly plausible, then the conclusion is only made weakly plausible by the argument.

However, in a convergent argument, each premise is a separate line of evidence, independent of the other premises. Therefore the conclusion is made as plausible as the most plausible premise, if the argument is valid. This principle is illustrated in the following example.

Case 5: Virgil said sincerely that there is a fire in the University.

Vanessa said sincerely that there is a fire in the University.

Therefore, there is a fire in the University.

This example is a convergent argument, for each premise individually constitutes a plausible argument for the conclusion without requiring the support of the other premise. Now let us suppose that Virgil is a highly reliable source on the subject of the fire in the University, and that Vanessa is a less reliable source. Suppose, in other words, that the first premise is highly plausible, but the second premise is only slightly plausible. What plausibility value should we assign to the conclusion? Clearly, we can infer that the conclusion is highly plausible, that it is at least as plausible as the first premise.

In short, the new rule is the following.

\section{PLAUSIBILITY RULE FOR CONVER- GENT ARGUMENTS:}

In a convergent argument, the conclusion is at least as plausible as the most plausible premise.

This rule then contrasts with the case of the linked argument, where the conclusion is assigned a plausibility value at least as great as the least plausible premise.

A complication is introduced through the fact that linked and convergent arguments can be combined, as below.

\section{Case 6: (1) A passerby reported smoke coming from the University.}

(2) If a passerby reported smoke coming from the University, then there is a fire in the University.

(3) The Fire Chief reported a fire in the University.

(4) If the Fire Chief reported a fire in the University, there is a fire in the University.

(5) Therefore, there is a fire in the University.

This example is a case of two linked arguments joined together in a convergent argument, as shown below.

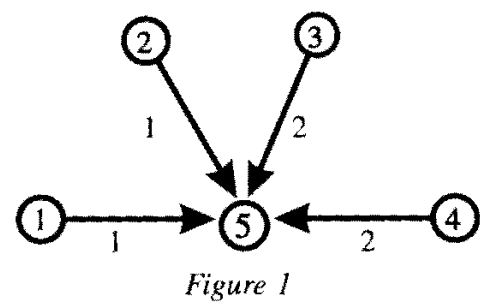

In this case, the second linked argument is stronger than the first. Therefore, the plausibility of the conclusion, (5), should be at least as high as that of the least plausible premise of the argument that has (3) and (4) as premises. 
To illustrate the point more clearly, let us presume that plausibility values can be assigned to each premise as follows. Values range between 0 and 1 , where 0 is the lowest plausibility a proposition can have and 1 is the highest plausibility.

$$
\begin{aligned}
\text { Case } 7 \text { :plaus }(1) & =.9 \text { (very highly plausible) } \\
\text { plaus }(2) & =.2 \text { (slightly plausible) } \\
\text { plaus }(3) & =.6 \text { (fairly plausible) } \\
\text { plaus }(4) & =.8 \text { (highly plausible) }
\end{aligned}
$$

The problem with this case is that if we straightforwardly apply the plausibility rule for convergent arguments given above, we would assign a value of .9 to the conclusion that there is a fire in the University. But this would be erroneous, since the very highly plausible premise (1) is linked to the premise (2), which is only slightly plausible. Hence the plausibility rule for convergent arguments must be modified to deal with this type of case. What must be done is to combine the least plausible premise rule with the plausibility rule for convergent arguments, in order to have a more generally applicable rule of plausible inference.

In the example above, clearly we need to consider each convergent argument separately, and pick the strongest one. But since each is a linked argument, the strongest will be the one with the highest least plausible premise. We have two convergent arguments to select from, with plausibility value given below.

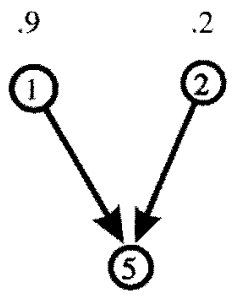

Figure 2

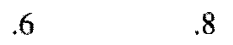

Using the least plausible premise rule, it is concluded that the linked argument on the right is the strongest, because its least plausible premise has a value of .6 , which is greater than the value of the least plausible premise of the other linked argument (.2). We conclude that the plausibility value of (5) is at least as great as that of (3), namely .6 (fairly plausible).
The general rule below covers cases where linked arguments are combined into convergent arguments.

\section{MAXMIN RULE:}

Collect together the values of the least plausible premises of all the linked arguments, and then pick the maximum of all these minimum values, for every conver. gent argument.

Even the maxmin rule above turns out to be oversimplified in certain respects, because it is possible to have various kinds of combinations of linked and convergent arguments in serial sequences.

A serial argument requires a successive readjustment of plausibility values. Suppose the initial values given a serial argument of the form ' $\mathrm{A} \rightarrow \mathrm{B} \rightarrow \mathrm{C}$ ' are the following: $\mathrm{A}=.6, \mathrm{~B}=.5, \mathrm{C}=.3$. First, $\mathrm{B}$ is adjusted upwards to a value of .6 . This evaluation follows the rule for single premised arguments where no other lines of argument lead in to the conclusion. The value of the conclusion is adjusted upwards to match that of the premise. Similarly, the value of $\mathrm{C}$ is then adjusted upwards to 6 .

Another type of case that can occur is illustrated by the following example, modelled by figure 3 . First, $C$ needs to be adjusted upwards to a plausibility value of .6 , in virtue of the least plausible premise rule for the linked argument. Then $E$ has to be adjusted upwards to .6 , in virtue of the rule for convergent arguments (maximum value).

\section{SERIALLY COMBINED LINKED AND CONVERGENT ARGUMENT}

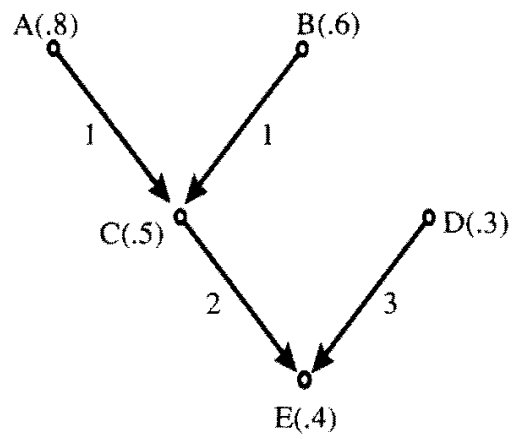

Figure 3 
To account for these complications, the MAXMIN RULE needs to be stated in a more general way, as follows.

\section{MAXMIN RULE:}

\begin{abstract}
Scan over the whole graph of the argument, starting at the initial premises (premises that have no lines of argument leading in to them) and adjust the values at the nodes upwards at each step, where required. according to the apppropriate rule, depending on whether that step is a linked argument or a convergent argument. For linked arguments, take minimum values of premises. For convergent arguments, take maximum values of premises.
\end{abstract}

The use of this new MAXMIN RULE is straightforward as applied to the method of using graphs to diagram complex sequences of argumentation in Walton and Batten (1984). Once a plausibility value has been assigned to each premise or conclusion, the appropriate adjustments are then made, using the MAXMIN RULE.

The basic thing to remember is the distinction between linked and convergent arguments. A convergent argument represents the idea of "new evidence" or a new line of argument that is independent of the previous premises of an argument. Convergent arguments do not follow the least plausible premise rule, because we are dealing with two "separate" arguments for the same conclusion, and this calls for a different kind of defending and questioning strategy.

Despite this exception, the least plausible premise rule still states a basic truth about plausible reasoning. Because plausible inference is inherently fallible, where premises are linked, the least value is taken.

\section{Might-Conditionals}

In the example from Intelliware (1986) presented in section 1 above, we saw how might-conditionals are dealt with in inference rules for inexact inferences in
AI: the product rule tells us to multiply the plausibility value (certainty factor) of the premise by that of the conditional (rule). This product rule is consistent with the basic philosophy behind plausible reasoning. Since the expert or source of information could be as wrong about a conditional as about a premise or simple proposition ( $\mathrm{fact}$ ), the plausibility of the conclusion drawn using that conditional as a rule of inference should be no greater than the least plausible of the premise and the conditional.

This general approach suggests the following rule: if a rule (conditional) and a fact (premise in a knowledge base) are combined to generate a conclusion, the plausibility value of the conclusion should be no greater than the lesser value of the pair of values given for the rule and the fact. The product rule also preserves the intent of this type of rule as well, however. For where the values combined are fractions between zero and one, their product will always be less than either value, taken singly. Indeed, the product rule is even more conservative, because it tends to lower the lower value. Let us call the first rule above the reduction rule, as opposed to the product rule.

The reduction rule, in effect, treats the rule of inference (conditional) as another value that needs to be factored in like a premise in the argument. This approach can be summed up in a new type of rule that allows values for might-conditionals to be counted in, even where the value is less than one.

\section{MAXMIN MIGHT RULE:}

\begin{abstract}
Rules of inference are to be assigned numerical plausibility values in arguments and counted in at the last stage of plausibility adjustment by being treated as a premise linked to the argument.
\end{abstract}

For example, suppose we have a linked argument with values as given below for the two premises, and the rule of inference is given a value of 4 . 


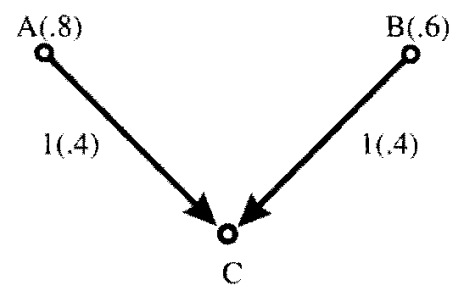

Figure 4

Now the number of the inference marked on the arc is given a number, representing its plausibility value. By the MAXMIN RULE, the least plausible premise, which has a value of .6 , should indicate an upward adjustment of the value of $\mathrm{C}$ to .6 as well, if it was less than that before. However, applying the more general MAXMIN MIGHT RULE, the value of $C$ would be adjusted upwards only to .4 , because that is the plausibility value of the rule of inference, 1 .

By contrast, the product rule would entail multiplying all three values $(.8 * .6 *$ .4) which would yield a final plausibility value of .2 for the conclusion. The product rule method generally tends to give a lower value for a conclusion than the reduction rule method.

What happens in the case of a convergent argument? This eventuality appears to be covered by a Rule for Combining Evidence given in Intelliware (1986, Main Menu, Inexact Inference, p. 8):

Suppose there are two rules which support a hypothesis. If $A$ and $B$ are the CF's obtained from these two rules, the combined certainty, Combine $(A, B)$ is defined as:

Combine $(A, B)=A+B-(A * B)$.

The following example (Intelliware, 1986 , p. 9) illustrates the use of the Rule for Combining Evidence with a case where there are two rules with values of .6 and .8 .

Case 8:

Rule 1:

Stock 12 is volatile

IF

Stock 12 is hightech $\quad \mathrm{CF}=0.90$ AND

Stock 12 is in demand

$\mathrm{CF}=0.60$

Evaluate Rule 1:

$\mathrm{CF}($ Rule 1$)=\operatorname{Min}(0.90,0.60) * 0.60=0.36$
Rule 2:

$$
\mathrm{CF}=0.70
$$

Stock 12 is volatile

IF

Stock 12 is a new issue $\quad \mathrm{CF}=0.80$

OR

Stock 12 is heavily traded $\quad \mathrm{CF}=0.40$

Evaluate Rule 2:

$\mathrm{CF}($ Rule 2) $=\operatorname{Max}(0.80,0.40) * 0.70=$ 0.56

Combine Evidence:

$\mathrm{CF}($ Stock 12 is volatile $)=0.36+0.56-$

$(0.36 * 0.56)=0.72$

In effect, the Rule for Combining Evidence appears to be a way of dealing with convergent arguments, at least in those cases where there are no premises in the one inference that are dependent on any premises in the other inference. Such is the case, it appears, in the example above, where each line of inference seems to be meant as an independent line of argument for the same conclusion (Stock 12 is volatile). However, it need not be so in every instance. In some cases where the Rule for Combining Evidence could be applied, some premises in the one inference could be dependent on, or even identical to, some premises in the other inference. By failing to make this distinction, the Rule for Combining Evidence is inadequate to deal with the need to distinguish between linked and convergent sub-arguments in a structure of argumentation.

The problem in cases of combined argumentation like the type of case confronted by the Rule for Combining Evidence is whether each line of argument is dependent on the other or not. These two kinds of cases need to be treated differently. One possibility is the case of two linked arguments combined to create a convergent argument at the macro level. This type of case is illustrated by the figure on the left below. Another quite different type of case is the one where two linked arguments are linked together by a third sub-argument. This type of case is illus- 
trated by the structure on the right in figure 5. The case on the left represents two linked arguments combined as a convergent argument. The case on the right represents two linked arguments, combined as a linked argument for a conclusion. Whether a product-style rule is applied, or a reduction-style rule is applied, in principle, each of these types of cases should be treated differently. The Rule for Combining Evidence appears to refer to the type of case pictured on the left, where each linked argument is a new line of evidence for the common conclusion. But the situation on the right could also possibly be covered by the same rule, and that is a problem.

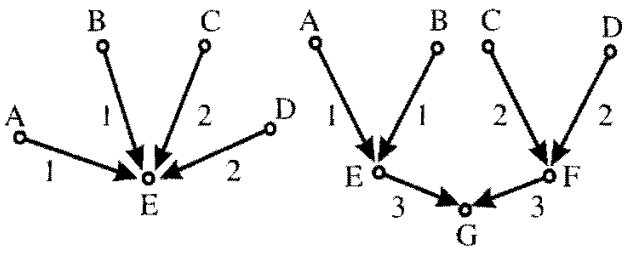

Figure 5

From a reduction rule perspective, the solution is given straightforwardly by the MAXMIN RULE. In the case on the right, the least value is chosen from the least values in each sub-argument. In the case on the left, the greater of the pair of least values is chosen. We will not propose a modified product-style rule to reflect the distinction between linked and convergent arguments. It suffices to comment that $\mathrm{AI}$ should look to taking account of this distinction in combined evidence rules.

\section{Applying the More Plausible Premises Rule}

There are many purposes of argument, but one stands out, especially in a critical discussion. A primary goal of any reasoned persuasion type of dialogue is for a proponent arguer to persuade or convince a recipient (respondent) arguer, by proving the proponent arguer's conclusion from the recipient arguer's premises. This means that to find a successful or useful argument, the proponent arguer must find premises that are relatively plausible to the respondent of the argument. And indeed, to be useful, an argument must have at least some, or even all premises that are more plausible for the recipient than the conclusion of the argument which the recipient doubts, or is reluctant to accept.

This requirement must be tempered by qualifications, however. If the argument is a linked argument, then each premise must be more plausible than the conclusion, in order for the argument to be useful in persuasion. But if the argument is convergent, it may be that only one of the premises, or perhaps some subset of the premises, needs to be more plausible. How the more plausible premises requirement is implemented will depend on the structure of the argument revealed by its argument reconstruction and diagram.

Another important qualification is that, at the local level, an argument may not need to have premises that are immediately more plausible. This is because adjustments of plausibility, according to MAXMIN RULE, may take place over a longer sequence of argumentation which may not yet be complete. What is required, then, is for an argument to show some promise or capability of leading to other premises that are more plausible. Any argument where this evidential route, through the premises, to further premises that may be more plausible, is "choked off" will fail the evidential priority requirement. And of course, an argument that commits the fallacy of begging the question is just such a case in point. Where an evidential route is left open so that subsequent argumentation could potentially lead to confirmation that the premises are more plausible to a degree useful to meet burden of proof, no allegation of the criticism that the argument begs the question arises.

For example, suppose that Ted is a biology student who states to Eva, his biology professor, that he finds it hard to 
believe that a whale is a mammal. After all, Ted says, "It looks like a fish." Ted asks Eva, "Can you prove to me that the whale is a mammal?" Eva replies with the following argument.

Case 9: If an animal suckles its young, then that animal is a mammal. The whale is an animal that suckles its young. Therefore, the whale is a mammal.

This linked argument is deductively valid, but what makes it useful as an argument to help to persuade Ted of the acceptability of its conclusion is that its premises are open to being proved to Ted. If the premises are immediately plausible to Ted, then that is the end of the argument. If they are not, then Eva can go on to supply further arguments for any premise questioned by Ted, in response to his critical questions.

Suppose Ted still maintains that he cannot bring himself to accept the first premise, because he does not find it plausible. Then Eva might respond with a further argument for this first premise. She might reply: "That is the accepted criterion for classification as a mammal in biology." Since Eva is herself a professor of biology, her argument here is a form of appeal to expertise (here in a pedagogical context of dialogue). An appeal to expertise can be a reasonable form of argument in some cases, and let us presume that, in this case, Ted finds the argument plausible, and has no objections to it. If Ted now finds the first premise of the argument above plausible, and already finds the other premise plausible (that the whale is an animal that suckles its young), then Ted will, or should, find the conclusion plausible as well.

The danger of the appeal to authority as a type of argument is that it can be pressed ahead too dogmatically or assertively as a tactic to block off critical questioning, turning into a fallacious argumentum ad verecundiam. ${ }^{7} \mathrm{But}$ in this case, no such fallacy needs to have been committed by Eva. For it is open to Ted, as a good biology student, to check up on Eva's claims. He can go to the library and check to see whether in fact there is evidence to confirm the premise that whales suckle their young. Or he can check studies on taxonomy to confirm the criteria for classifying an animal as a mammal. Provided Eva's argument has left these avenues open, it should not be criticized for convening or interfering with the implementation of the kind of plausibility requirement studied in section five.

The general pattern of Eva's use of the argument to alter Ted's commitments is clear. Because Ted could be convinced that the premises are plausible, and because the argument itself had a structure that enabled plausibility to be transferred to the conclusion (indeed, it was deductively valid, in this case), Ted could be persuaded by the argument to accept the conclusion as a plausible proposition. This pattern of argument leads to the following working implementation of the more plausible premises rule as applicable to cases where a critic has the job of evaluating whether an argument begs the question or not.

\section{MORE PLAUSIBLE PREMISES RULE:}

If an argument is to be capable of meeting the requirement of evidential priority which is to make it a useful or potentially successful argument relative to a critical dis. cussion, then (a) the premises must be more plausible than the conclusion, or (b) routes of further argument to the premises must be open so that, through further argument, the premises could be shown to be more plausible than the conclusion, as the dialogue continues.

It is important to note that applying this rule depends on the argument reconstruction. If the argument is linked, then each premise must be more plausible (actually or potentially). But if it is convergent, only one more plausible line of argument needs to be open.

It should be pointed out that two versions of the more plausible rule are open to consideration. The version above is the 
weaker version. The stronger version deletes clause (b) above, retaining only clause (a). No doubt, many would prefer to adopt the stronger version, instead of the weaker version proposed above. The issue of which version is chosen has highly significant implications for any analysis of the fallacy of begging the question. ${ }^{8}$

The problem with the stronger version is that it leaves the proponent of a thesis no room to develop an argument. If he asks his respondent to tentatively accept a premise, in order to open up a line of argumentation, even though this premise is not (immediately) more plausible than the conclusion to be proved, the respondent can at once criticize his argument for committing the fallacy of begging the question. It is for this reason that the weaker version of the more plausible premises rule is preferable in some cases. However, once a critical discussion has been properly closed, and all the relevant arguments on both sides have been considered, the strong version of the more plausible premises could be the more appropriate version. But in fact, criticisms are often made in the middle (argumentation stages) of a dialogue. Hence the more dynamic (weaker) version of the more plausible premises rule is more generally applicable at the argumentation stage of a critical discussion.

The reason that the more plausible premises rule is appropriate in this case is that Ted has expressed frank doubts that the conclusion is plausible. Therefore, in order to overcome these doubts, Eva will have to find premises that are more plausible than the degree of plausibility that Ted initially attaches to the conclusion. This case is not a compound dispute, in the sense of van Eemeren and Grootendorst (1984), as far as we know from the corpus of the dialogue, at any rate. Ted, in other words, does not enter into the argument with the thesis that whales are not mammals. He has only expressed doubts about the plausibility of this proposition.
The more plausible premises rule is not a requirement of every context of argument. In some cases, it is clearly obligatory. In other cases, we may not know whether it is an appropriate requirement or not, because we simply do not know enough about the context of dialogue from the given corpus of the argument. And in other cases, it can be evident that it is not a requirement.

\section{Arguments that are Useless for Persuading}

What makes an argument useful for the purpose of reasonable persuasion is that the plausibility value of the premises should be (at least potentially) greater than that of the conclusion, from the point of view of the respondent to whom the argument was addressed. The rationale behind this requirement is simple. The respondent in a critical discussion is disinclined to accept the conclusion of an argument presented to him by the proponent. The respondent needs to be convinced. How to convince him? The usual way is for the proponent of the argument to present premises that the respondent is already committed to, or, at any rate, premises that he can be brought to accept, because he can find them plausible. Then the proponent can use these premises, in arguments that have conclusions that the respondent can be driven (persuaded) to accept, by means of these arguments.

What we are talking about here is not just the logical form or semantic structure of these arguments, per se. We are talking about how such arguments can be used in order to fulfill goals of dialogue, e.g. to persuade a respondent to accept a conclusion he is initially inclined to be doubtful about. Arguments that fail the more plausible premises requirement are not faulty or open to criticism because they are deductively invalid, or because they fail to have a semantically valid form of argument. They are faulted 
because, even if they are deductively valid, they are useless to persuade a doubter.

The basis of criticism in a critical discussion is not always that the argument is formally invalid. Rather, a common and legitimate type of criticism is that the argument is not useful for the purpose it was supposed to have in the critical discussion designed to resolve a conflict of opinions. To resolve such a difference of opinions, plausible reasoning must be brought to bear through arguments that can be useful to change a respondent's opinions on an issue.

The two basic configurations of argumentation that are useful for the purpose of reasoned persuasion are the linked argument and the convergent argument. These are pragmatic structures of argumentation, and the distinction between them is therefore best seen as relative to a context of reasoned dialogue. Here, we have been primarily concerned with critical discussion, although, to be sure, other contexts of argumentation could be important as well.

In a critical discussion, the distinction between the linked and convergent structures of argumentation is to be drawn in tactical terms of successful attack and defence. In the linked argument, a successful attack or questioning of the argument implies that the whole argument "falls down" (is refuted). By contrast, in the convergent argument, a successful attack still leaves open the possibility of a successful rebuttal. This way of putting the distinction in terms of attack and defence is fruitful and appropriate in persuasion dialogue, because of the designated rules of the two participants in this type of dialogue. The proponent has the burden of proof-he must persuade the respondent, using plausible premises, in order to win the game. His argument has to "move forward" from the premises to the conclusion. The respondent-the person to whom the argument is directed in dialogue-has the burden or rule of questioning (resisting) the argument. If he fails to do this successfully, the argument will go forward and carry the weight of presumption, by default. Whether the argument is good or bad, defensible or fallacious, and so forth (positively or negatively evaluated) depends on the shifting back and forth of these burdens. Therefore, ultimately the criterion of how the argument is to be evaluated can be put in terms of available attacks and defences in a context of dialogue.
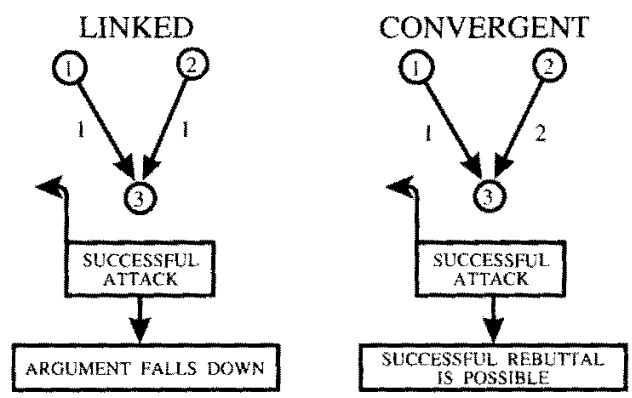

Figure 6

The critic's strategy in a linked argument should be to attack the weakest premise. The analogy is to the attackers of a medieval castle. The attacking force seeks out the weakest point in the wall. The defenders, consequently, must concentrate their forces on that point as well, trying to patch up the weak spot as strongly as necessary to repel the attack.

In a convergent argument, however, the critic's strategy should be to attack the strongest premises first. Once again, the defender must match the point of attack. If one side is not plausible or strong, he must go to the other side, and try to build up that defence. The analogy here is not that of defending a fort. It is like a two-pronged (or multiple-pronged, in the general case) attack, where there are two separate columns of attacking forces. When one column is met with a counter-attack that overwhelms it, the appropriate tactic must be to press ahead with the other stronger column, in the hope of breaking up the counter-attack. If one line of effort is not working for the defender, his best tactic is to go to the other one. In general, his best strategy is to back up his strongest line of argument as fully as possible. If another line appears weak, it 
can be abandoned without losing the overall struggle.

Plausibility rules for divergent and serial argumentation can also be formulated. In a divergent argument, you can conclude to either conclusion (2) or conclusion (3), below.

\section{DIVERGENT ARGUMENT}

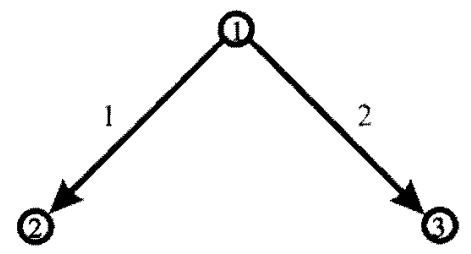

Figure 7

Hence, in this kind of argumentation, the plausibility value of both 2 and 3 should be adjusted upwards to the value of (1), if (2) and (3) are not already at that value or higher.

With serial argumentation, the MAXMIN RULE is operative, because everything depends on whether the links in the chain of argumentation are linked or convergent. But generally, the longer the chain of argument for a conclusion, the more escape routes and openings for questioning there will be for a critic to find. Therefore, the basic principle governing serial argumentation in persuasion dialogue was enunciated by Windes and Hastings (1965, p. 218) as follows: "[The proponent] should begin the chain of proof at the most advanced evidence which the audience will accept and move to the proposition [the conclusion] from there." In a serial argument the values are adjusted upwards sequentially.

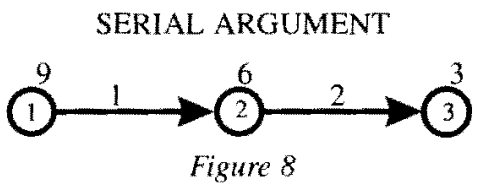

In the argument in figure 8 , for example, if both steps 1 and 2 are plausible and complete arguments for the conclusions (2) and (3) respectively, then the required plausi- bility adjustments are as follows. First, the value of (2) is raised to 9 , to meet the value of 1 . Then, in a second phase of adjustment, the value of (3) is raised to 6 , to meet the value of (2). But then a third phase of adjustment is also required-the value of (3) must be raised again to meet the new value of (2), namely to 9. Thus serial argumentation requires a whole series of adjustments, as far along a chain of argumentation as is required to meet all adjustments. The problem is that arguments in persuasion dialogues are not one-step affairs. A respondent must often give a proponent of an argument "room to argue," meaning that it may not always be reasonable to immediately require premises that are more plausible. In some cases, a premise could be acceptable, at least provisionally, if it shows promise of leading to other premises (from which it follows) that are more plausible. In other words, it is not the immediate premises of an inference, but the ultimate premises that are required to be more plausible, if the chain of argumentation based on those premises is to be successful in persuasion regarding a doubtful matter.

Hamblin's reorientation of the problem shows that we need to evaluate the worth of premises in a persuasion dialogue in relation to how these premises can ultimately stand up to critical scrutiny by the respondent they were advanced to convince. And this, in turn, indicates the importance of the distinction between linked and convergent arguments. It will be recalled that, according to van Eemeren and Grootendorst (1984, p. 91), the crucial difference between linked and convergent argumentation turned on the sequences of the respondent's calling the argument into question. In the linked argument, the proponent has to defend all his premises, whereas in the convergent argument, the proponent need only defend one premise as plausible in order to meet the goal of convincing the respondent. The new rules proposed above fit these requirements of 
the use of reasoning to rationally persuade a respondent in a critical discussion in order (ultimately) to resolve a conflict of opinions.

Generally, it seems appropriate to have different kinds of rules of plausible reasoning for different types of dialogue in which argumentation occurs. However, it is the contention of this paper that Rescher's "least plausible premise" approach is suitable to provide the basis for a set of rules appropriate for the critical discussion as a type of dialogue. However, these rules require the additions and modifications proposed above, in order to fit this context of the use of argumentation.

\section{Notes}

1 Research for this paper was supported by three awards: (1) a Killam Research Fellowship from the Canada Council, (2) a Fellowship from the Netherlands Institute for Advanced Study in the Humanities and Social Sciences, and (3) a Research Grant from the Social Sciences and Humanities Research Council of Canada.

2 Walton (1990, chapter eight).

3 Walton (1989, chapter seven).

4 See Walton (1990, pp. 74-77).
5 For further background on how plausible reasoning fits into Rescher's general conception of reasoned argument, see Rescher (1977) and (1988).

6 On this notation for argument diagramming, see Walton and Batten (1984).

7 See Walton (1989, chapter seven).

8 See Walton (1991).

9 Van Eemeren and Grootendorst (1984, p. 80).

\section{References}

Ivan Bratko, Prolog Programming for Artificial Intelligence, Reading, Mass., AddisonWesley, 1986.

Richard Forsyth, Expert Systems, London, Chapman and Hall, 1984.

Charles L. Hamblin, Fallacies, London, Methuen, 1970.

Intelliware, Experteach (Software and Manual), Intelligence Ware, Inc., Los Angeles, 1986.

Nicholas Rescher, Plausible Reasoning, AssenAmsterdam, Van Gorcum, 1976.

Nicholas Rescher, Dialectics, Albany, State University of New York Press, 1977.

Nicholas Rescher, Rationality, Oxford, Oxford University Press, 1988.

Frans H. van Eemeren and Rob Grootendorst, Speech Acts in Argumentative Discussions, Dordrecht and Cinnaminson, Foris Publications, 1984.
Douglas N. Walton, Informal Logic, Cambridge, Cambridge University Press, 1989.

Douglas N. Walton, Practical Reasoning, Savage, Maryland, Rowman and Littlefield, 1990.

Douglas N. Walton, Begging the Question, New York, Greenwood Press, 1991.

Douglas N. Walton, Plausible Argument in Everyday Conversation, Albany, SUNY Press, 1992.

Douglas N. Walton and Lynn M. Batten, "Games, Graphs and Circular Arguments," Logique et Analyse, 106, 1984, 133-64.

Russel R. Windes and Arthur Hastings, ArgumenIation \& Advocacy, New York, Random House, 1965

DOUGLAS WALTON

DEPARTMENT OF PHILOSOPHY

UNIVERSITY OF WINNIPEG

WINNIPEG, MANITOBA R3B $2 E 9$ 